\title{
Left Atrial Function by Two-Dimensional Speckle Tracking Echocardiography in Patients with Severe Rheumatic Mitral Stenosis and Pulmonary Hypertension
}

\author{
VIMAL MEHTA ${ }^{1}$, Dhanjibhai Chaudhari ${ }^{1}$, Pratishtha Mehra ${ }^{1}$, Sudhanshu Mahajan ${ }^{1}$, \\ Jamal Yusuf ${ }^{1}$, Safal Safal ${ }^{1}$, Sanjeev Kathuria ${ }^{1}$, and Saibal Mukhopadhyay ${ }^{1}$ \\ ${ }^{1}$ GB Pant Hospital
}

May 11, 2021

\begin{abstract}
Aim: The aim of this study was to assess the left atrial (LA) function in severe rheumatic mitral stenosis (MS) patients using two-dimensional speckle tracking echocardiography (STE) and its correlation with clinical symptoms and echocardiography parameters. Methods: A total of 120 subjects (80 patients with isolated severe MS [mitral valve area (MVA) [?]1.5 cm2] in sinus rhythm and 40 healthy controls) underwent comprehensive echocardiography including STE for assessment of LA strain [reservoir strain (LASr), conduit strain (LAScd) and contractile strain (LASct)]. Results: The mean MVA in cases was $0.93 \pm$ $0.21 \mathrm{~cm} 2$. The mean values of LASr $(14.73 \pm 8.59 \%)$, LAScd $(-7.61 \pm 4.47 \%)$ and LASct $(-7.16 \pm 5.15 \%)$ among cases were significantly less $(\mathrm{p}<0.001)$ when compared to controls where the values were $44.11 \pm 10.44 \%,-32.45 \pm 7.63 \%,-11.85 \pm 6.77 \%$ respectively. Thus the compensatory LA contractile function was also compromised. The New York Heart Association (NYHA) class III, II and I dyspnea was present in 37 (46.25\%), 38 (47.5\%) and 5 (6.25\%) subjects respectively. All the three LA strain parameters showed a trend towards decline with increase in severity of MS, increase in LA size, increase in mean and peak diastolic transmitral gradients and with higher NYHA functional class. Conclusion: Left atrial dysfunction is common in severe rheumatic MS as suggested by severely reduced LA reservoir, conduit and contractile strain. Early and timely intervention in these patients irrespective of NYHA functional class is advocated as it may likely improve the LA function and avoid clinical deterioration.
\end{abstract}

\section{Introduction}

Rheumatic heart disease (RHD) is endemic in developing countries, where it remains the second most common cause of cardiovascular morbidity and mortality after atherosclerotic cardiovascular disease Mitral valve is the most commonly involved valve being afflicted in $60.2 \%$ patients, with mitral stenosis (MS) being the most common valve lesion in chronic RHD. MS causes obstruction to left ventricular (LV) diastolic filling, leading to morphological and functional changes in left atrium (LA) as a result of LA pressure overload. The elevated LA pressure is transmitted back to pulmonary circulation resulting in exertional dyspnea and ultimately leads to development of post-capillary pulmonary hypertension. LA compliance is an important determinant of LA pressure as different LA pressures are recorded in different subjects despite similar mitral valve areas (MVA). ${ }^{4}$ Chronic LA pressure overload leads to atrial muscle bundle disorganization and fibrosis resulting in both atrial stiffness and atrial reservoir dysfunction. ${ }^{5}$ The left atrial dysfunction may be detected and quantified by deformation imaging i.e. speckle tracking echocardiography (STE). STE is a reliable and effective tool for evaluating LA function.

LA dysfunction has been reported in patients with rheumatic MS. ${ }^{10}$ However most studies have either assessed global LA strain, reservoir or conduit strain values with contractile strain being reported only in mild to moderate MS. ${ }^{10,11}$ Only very limited data is available regarding all three strain parameters in patients 
with severe MS. In the present study we assessed LA function (reservoir, conduit strain and contractile strain) by two-dimensional speckle tracking echocardiography and its correlation with clinical symptoms and echocardiography parameters in patients with isolated severe MS with pulmonary hypertension and healthy controls.

\section{Material and methods}

This study was a comparative cross-sectional study conducted at a tertiary care hospital in North India in which 120 subjects including 80 patients with isolated severe MS (MVA [?]1.5 $\mathrm{cm}^{2}$ ) with pulmonary hypertension between 18-40 years of age who were in sinus rhythm and 40 age matched healthy controls were enrolled from cardiology outpatient department. Patients with moderate to severe mitral regurgitation or other significant valve involvement and co-morbidities like hypertension, diabetes mellitus, coronary artery disease, overt LV systolic dysfunction were excluded. Philips EpiQ 7C echocardiography system with $2.4 \mathrm{MHz}$ transducer was used for recording echocardiographic images. The study was carried out after prior approval from the institutional ethical committee and written informed consent was obtained from all the participants.

For all subjects, standard two-dimensional (2D), M-mode and Doppler echocardiograms were obtained according to the American Society of Echocardiography guidelines. ${ }^{11}$ Baseline measurements included were LA dimension, LV internal dimension in diastole $\left(\mathrm{LVID}_{\mathrm{d}}\right)$ and in systole $\left(\mathrm{LVID}_{\mathrm{s}}\right)$ and $\mathrm{LV}$ ejection fraction (LVEF). MVA [?]1.5 $\mathrm{cm}^{2}$ (by planimetry) was considered to define severe MS and MVA $<1.0 \mathrm{~cm}^{2}$ defined very severe MS. ${ }^{13}$ Peak diastolic gradient (PG) and mean diastolic gradient (MG) across the mitral valve was calculated by continuous wave (CW) Doppler tracing. Apical four chamber (A4C) and apical two chamber (A2C) views were recorded in all the patients. Right ventricle systolic pressure (RVSP) was measured from tricuspid regurgitation jet velocity by $\mathrm{CW}$ Doppler after ruling out primary involvement of tricuspid and pulmonary valve. ${ }^{14}$ Three consecutive cardiac cycles were recorded and averaged.

Pulmonary hypertension ( $\mathrm{PH}$ ) was graded based on peak RVSP as mild 36-45 mm Hg, moderate 46-60 $\mathrm{mm} \mathrm{Hg}$ and severe if $>60 \mathrm{~mm} \mathrm{Hg} .{ }^{15}$ Patients were classified according to the New York Heart Association (NYHA) functional classification. Patients in NYHA class IV were not included because all the patients recruited were from OPD visits and were ambulatory.

\section{Left atrial two dimensional speckle tracking echocardiography}

LA images were analyzed offline via QLAB 13 Philips software for speckle tracking as depicted in Figure 1. LA deformation is a cyclical process, which can be sub-divided into three phases: a) Reservoir phase: starts at the end of ventricular diastole (mitral valve closure) and continues until mitral valve opening. It encompasses the time of LV isovolumic contraction, ejection and isovolumic relaxation, b) Conduit phase: occurs from the time of mitral valve opening through diastasis until the onset of LA contraction, c) Contraction phase: occurs from the onset of LA contraction until the end of ventricular diastole (mitral valve closure). For offline analysis of LA function, cardiac cycle was gated with ECG and kept from end-diastole to end-diastole with zero reference point taken as peak of $\mathrm{R}$ wave. ${ }^{16}$ After selection of appropriate image and ECG gating of cardiac cycle, software automatically traces the endocardial border of LA while manual adjustments are done to exclude pulmonary veins and LA appendage.

\section{Statistical analysis}

Epi Info version 7.1.1 software was used for the analysis of the data. Continuous data were presented as mean with standard deviation and compared by t-test. Continuous data of more than two groups were compared by ANOVA test. Categorical data were presented as frequency and percentage and compared using a chisquare test. $\mathrm{P}$ value $<0.05$ was considered as significant. To investigate for inter-observer variability for LA strain, analysis of 10 random subjects was done by two independent investigators who were blinded to the clinical data. For intra-observer variability, repeat offline LA strain estimation was done at $5 \pm 2$ days later in 10 randomly selected patients. The interclass correlation coefficients (ICCs) were calculated with point estimates and $95 \%$ confidence intervals (CIs) being reported.

\section{Results}


We enrolled 120 subjects including 80 cases and 40 controls in our study. There were 57 females and 23 males amongst cases while among control population there were 15 females and 25 males. Table 1 shows the baseline characteristics and various echocardiographic parameters among study subjects. The mean LA size among cases was $4.67 \pm 0.65 \mathrm{~cm}$ and mean MVA was $0.93 \pm 0.21 \mathrm{~cm}^{2}$. Severe MS (MVA $1-1.5 \mathrm{~cm}^{2}$ ) was seen in $44(55 \%)$ subjects while very severe MS (MVA $\left.<1 \mathrm{~cm}^{2}\right)$ was seen in $36(45 \%)$ subjects. The mean RVSP in cases was $60.01 \pm 19.88 \mathrm{~mm} \mathrm{Hg}$ suggesting moderate to severe pulmonary hypertension. All three STE derived LA strain [reservoir strain $\left(\mathrm{LAS}_{\mathrm{r}}\right)$, conduit strain $\left(\mathrm{LAS}_{\mathrm{cd}}\right)$ and contractile strain $\left.\left(\mathrm{LAS}_{\mathrm{ct}}\right)\right]$ parameters were significantly reduced among cases $(\mathrm{p}<0.001)$ with mean values of $\operatorname{LAS}_{\mathrm{r}}(14.73 \pm 8.59 \%)$, $\mathrm{LAS}_{\mathrm{cd}}(-7.61 \pm 4.47 \%)$ and $\mathrm{LAS}_{\mathrm{ct}}(-7.16 \pm 5.15 \%)$ when compared to controls where the mean values were $44.11 \pm 10.44 \%,-32.45 \pm 7.63 \%,-11.85 \pm 6.77 \%$ respectively. The interclass correlation coefficient for LA strain measurement was 0.95 (95\% CI: 0.84-0.98) for inter-observer agreement and 0.97 (95\% CI: 0.94-0.99) for intra-observer agreement, indicating good inter-observer and intra-observer correlations.

The NYHA class III dyspnea was present in 37 (46.25\%) subjects, while thirty-eight (47.5\%) subjects had NYHA class II dyspnea followed by $5(6.25 \%)$ subjects who had NYHA class I dyspnea. The descriptive statistics associated with patients in different NYHA classes were analyzed both between the groups and within group by ANOVA (Table 2). There was no significant correlation between any echocardiography derived parameters as well as LA strain parameters between various NYHA classes except for MVA which was significantly lower in NYHA class III $\left(0.87 \pm 0.21 \mathrm{~cm}^{2}\right)$ when compared to NYHA class II $(0.97 \pm 0.2$ $\left.\mathrm{cm}^{2}\right)$ and I $\left(1.18 \pm 0.23 \mathrm{~cm}^{2}\right)[\mathrm{p}=0.004$ between all groups] and RVSP which was significantly higher in NYHA class III $(66.32 \pm 19.74 \mathrm{mmHg})$ when compared to class II $(55.03 \pm 18.47)[\mathrm{p}=0.01$ between NYHA class II and III]. All the three mean LA strain values (reservoir, conduit, contractile strain) were numerically lower in NYHA class III when compared to NYHA class I patients but did not reach statistically significant difference.

We divided cases into groups based on increasing mean diastolic transmitral gradient (four groups), increasing peak diastolic transmitral gradient (three groups), increasing left atrial size (four groups), increasing severity of pulmonary hypertension (four groups) and decreasing mitral valve area (two groups) to study their correlation with LA strain (Table 3). The numerical value of all three STE derived LA strain parameters showed a trend towards decline with decrease in MVA, increase in LA size and increase in severity of PH, increase in MG and PG. But none of the above correlations achieved statistical significance (Table 4).

\section{Discussion}

The main finding of our study is a significant decrease in all three left atrial strain parameters i.e. reservoir, conduit and contractile strain suggestive of severe LA dysfunction in patients with severe rheumatic MS when compared to healthy controls. It has been shown in a previous study by Mahfouz et al ${ }^{17}$ on 75 patients that the conduit and reservoir function are affected in mild MS while LA contractile strain is well preserved. The increased LA contractile function appears as a compensatory mechanism to counterbalance reduced LA reservoir and conduit function in mild MS as LV filling predominantly occurs in LA contractile phase in MS in contrast to normal filling pattern where LA filling predominantly occurs in the early conduit phase. ${ }^{18}$ However characterizing the various components of LA function in MS and in other disease states requires complex methodology. ${ }^{19}$ The speckle tracking echocardiography provides an opportunity to quantitatively characterize various components of LA function non-invasively. Therefore, our study population which comprised of patients with severe MS $(\mathrm{n}=44)$ and very severe MS $(\mathrm{n}=36)$ could explain the reduction in all three LA strain parameters suggesting advanced degree of LA dysfunction. Our study shows that in severe MS, LA contractile function is also compromised in addition to reservoir and conduit function. Another study by Demirkol et al on 52 asymptomatic MS patients also showed that LA reservoir and conduit strain was significantly reduced but the contractile strain was increased in MS patients when compared to control population. This could similarly be explained by difference in the characteristics of study population as in their study cohort ${ }^{10}$, MS patients had mean MVA by planimetry of $1.38 \pm 0.36 \mathrm{~cm}^{2}$ with mean diastolic transmitral gradient of $7.9 \pm 2.8 \mathrm{~mm} \mathrm{Hg}$ in contrast to our study where MS was more severe as mean MVA was $0.93 \pm 0.21 \mathrm{~cm}^{2}$ and mean transmitral gradient was $12.33 \pm 4.16 \mathrm{~mm} \mathrm{Hg}$ which could have compromised 
the contractile function.

93.75\%) subjects in our study were in NYHA functional class II and III. There was a non-significant trend of higher mean LA size, peak and mean diastolic transmitral gradients and RVSP with increasing NYHA functional class while mean MVA was significantly less in NYHA class III group when compared to class II and I group ( $p=0.004$ between all groups). There was trend towards stepwise decrease in LA reservoir, conduit and contractile strain with deteriorating NYHA functional class, however this did not reach statistical significance. Our results were different from a study by Chien et $\mathrm{al}^{20}$ on $69 \mathrm{MS}$ patients which showed positive correlation between atrial deformation and NYHA functional class. In their study cohort, the mean MVA by planimetry was $1.41 \pm 0.50 \mathrm{~cm}^{2}$ in contrast to our study where mean MVA was $0.93 \pm 0.21$ $\mathrm{cm}^{2}$ and all patients had severe MS and none with mild/moderate MS. Hence, LA strain parameters were markedly decreased in our study population of severe MS patients (suggesting LA dysfunction) making further numerical fall with deteriorating NYHA functional class inconsequential. Secondly, in their study ${ }^{20}$, atrial fibrillation (AF) patients constituted $57 \%$ of the study population, hence LA contractile strain was not reported. Their results of atrial deformation are based upon LA reservoir strain in addition to reservoir and conduit strain rate. In our study, we excluded AF patients and we systematically evaluated and analyzed all three LA strain parameters in our study cohort.

Mitral stenosis results in obstruction to LV filling resulting in LA pressure overload which leads to alteration in LA geometry and function with progressive interstitial fibrosis, dilatation and remodeling of LA, ultimately culminating in LA dysfunction. ${ }^{5}$ However, LA remodeling is at least partially reversible and mitral valve intervention in the form of balloon mitral valvotomy/surgery can relieve LA pressure overload, thereby reducing LA size and improving LA function leading to reverse remodeling. ${ }^{22}$

Our study has important clinical implication in that the patients with severe MS regardless of severity of NYHA functional class develop severe LA dysfunction which worsens with further decline in MVA. Therefore patients with severe MS should be subjected to early and timely BMV so as to improve their LA function. ${ }^{11,22}$ We also believe that intervention may have an impact on preventing these patients to develop atrial fibrillation (AF), RV dysfunction and improving their prognosis. In the study by Ancona et $\mathrm{al}^{23}$ the degree of reduced LA systolic strain in patients with rheumatic MS correlated not only with worse cardiovascular outcomes during 3-year follow-up but also was the most powerful predictor of new onset AF at 4-year follow-up. In our study population of severe mitral stenosis and pulmonary hypertension, marked LA dysfunction was present. The high RVSP also likely contributed to LA dysfunction as higher pulmonary artery pressures have been reported to have strong negative correlation with LA compliance. ${ }^{4}$ Vriz et $\mathrm{al}^{24}$ showed that reduced LA reservoir strain can predict development of RV impairment and AF in patients with severe MS better than transmitral gradients.

Finally, many factors could have contributed to this LA dysfunction including chronic LA pressure overload, LA fibrosis, adverse remodeling of LA, involvement of mitral apparatus in the rheumatic process, LV and RV dysfunction. In fact our group has recently shown that decrease in deformation of basal segments of LV is more compared to mid and apical LV segments suggesting rheumatic endocarditis and scarring extend from the mitral annulus to the surrounding basal LV myocardial segments. ${ }^{25}$

\section{Limitations}

The major limitation of our study is that we included only isolated severe MS patients and hence our results are not applicable to patients with multi-valvular disease or other valve disease. This was done so as to have a uniform study population as including subjects with regurgitant lesions would have resulted in volume overload of the left atrium thereby affecting the results. Secondly our results are not applicable to patients in $\mathrm{AF}$ as they were excluded. This was because the speckle tracking echocardiography is ideally for patients with regular heart rhythms as strain values are directly influenced by the length of diastole. Thirdly, image quality and different vendor machines as well different versions of software of the same vendor may affect the results. However, the same equipment and software were used to assess all study subjects thereby avoiding any inconsistency in measurements. Fourthly invasive cardiac catheterization was not done in our study. This 
was because two-dimensional echocardiography is standard technique to evaluate patients with rheumatic MS. Lastly we did not study the effect of BMV on LA strain parameters which requires further investigation.

\section{Conclusion}

The present study shows that marked left atrial dysfunction is seen in severe rheumatic MS irrespective of NYHA functional class as suggested by severely reduced left atrial reservoir, conduit and contractile strain. LA function deteriorated further non-significantly with increasing severity of MS (decreasing mitral valve area), increase in left atrial size and increase in mean and peak transmitral gradient. Hence, our study results suggests that early and timely intervention in severe MS patients irrespective of NYHA functional class should be done as it may likely improve the LA function and avoid clinical deterioration. Further BMV may retard the development of AF and RV dysfunction. This hypothesis however requires further study.

Conflicts of Interest : All the authors declare that they have no conflicts of interest with respect to the present submission.

\section{Author contributions}

Vimal Mehta: Conceptualization, Data collection, Investigation, Methodology, Formal analysis, Supervision, Writing- Review \& Editing- Original \& Final Draft, Approval of final manuscript. Dhanjibhai Chaudhari: Data collection, Formal analysis, Approval of final manuscript. Pratishtha Mehra: Conceptualization, Data collection, Investigation, Methodology, Formal analysis, Writing- Review \& Editing, Resources, Approval of final manuscript. Sudhanshu Mahajan: Data analysis, Writing- Review \& Editing- Original \& Final Draft, Approval of final manuscript. Jamal Yusuf: Validation, Review \& Editing, Approval of final manuscript. Safal: Critical intellectual inputs, Review \& Editing, Approval of final manuscript. Sanjeev Kathuria: Validation, Review \& Editing, Approval of final manuscript. Saibal Mukhopadhyay: Methodology, Resources, Approval of final manuscript.

\section{References}

1. Padmavati S. Rheumatic fever and rheumatic heart disease in India at the turn of the century. Indian Heart J. 2001;53:35-7.

2. Soler-Soler J, Galve E. Worldwide perspective of valve disease. Heart. 2000;83(6):721-5.

3. Manjunath CN, Srinivas P, Ravindranath KS, Dhanalakshmi C. Incidence and patterns of valvular heart disease in a tertiary care high-volume cardiac center: A single center experience. Indian Heart J. 2014;66(3):320-6.

4. Kapoor A, Kumar S, Shukla A, et al. Determinants of left atrial pressure in rheumatic mitral stenosis: role of left atrial compliance and "atrial stiffness". Indian Heart J. 2004;56:27-31.

5. Thiedemann KU, Ferrans VJ. Left atrial ultrastructure in mitral valvular disease. Am J Pathol. 1977;89:575-604.

6. Amundsen BH, Helle-Valle T, Edvardsen T, et al. Noninvasive myocardial strain measurement by speckle tracking echocardiography: Validation against sonomicrometry and tagged magnetic resonance imaging. J Am Coll Cardiol. 2006;47(4):789-93.

7. Perk G, Tunick PA, Kronzon I. Non-Doppler two-dimensional strain imaging by echocardiographyFrom technical considerations to clinical applications. J Am Soc Echocardiogr. 2007;20:234-43.

8. Ozdemir AO, Kaya CT, Ozdol C, et al. Two-dimensional longitudinal strain and strain rate imaging for assessing the right ventricular function in patients with mitral stenosis. Echocardiography. 2010;27(5):525-33.

9. Fontana A, Zambon A, Cesana F, Giannattasio C, Trocino G. Tissue Doppler, triplane echocardiography, and speckle tracking echocardiography: different ways of measuring longitudinal myocardial velocity and deformation parameters. A comparative clinical study. Echocardiography. 2012;29(4):42837.

10. Demirkol S, Kucuk U, Baysan O, et al. The impact of mitral stenosis on left atrial function assessed by two-dimensional speckle tracking echocardiography. Echocardiography. 2012;29:1064-70. 
11. Sravan K Reddy N, Ranjan Shetty K, Sudhakar Rao M, Sree Madhurya Reddy M. Strain imaging to assess early effects of successful percutaneous balloon mitral valvotomy on left atrium mechanics. Egypt Heart J. 2019;71(1):25.

12. Lang RM, Badano LP, Mor-Avi V, et al. Recommendations for cardiac chamber quantification by echocardiography in adults: an update from the American Society of Echocardiography and the European Association of Cardiovascular Imaging. Eur Heart J Cardiovasc Imaging. 2016;16:233-271.

13. Baumgartner H, Hung J, Bermejo J, et al. Echocardiographic assessment of valve stenosis: EAE/ASE recommendations for clinical practice. Eur J Echocardiogr. 2009;10:1-25.

14. Rudski LG, Lai WW, Afilalo J, et al. Guidelines for the echocardiographic assessment of the right heart in adults: a report from the American Society of Echocardiography endorsed by the European Association of Echocardiography, a registered branch of the European Society of Cardiology, and the Canadian Society of Echocardiography. J Am Soc Echocardiogr. 2010;23:685-713.

15. Mehra P, Mehta V, Sukhija R, et al. Pulmonary hypertension in left heart disease. Arch Med Sci. 2019;15(1):262-73.

16. Badano LP, Kolias TJ, Muraru D, et al. Standardization of left atrial, right ventricular, and right atrial deformation imaging using two-dimensional speckle tracking echocardiography: a consensus document of the EACVI/ASE/Industry Task Force to standardize deformation imaging. Eur Heart J Cardiovasc Imaging. 2018;19:591-600.

17. Mahfouz RA, Gouda M, Abdelhamed M. Relation between left atrial strain and exercise tolerance in patients with mild mitral stenosis: An insight from 2D speckle-tracking echocardiography. Echocardiography. 2020;37(9):1406-12.

18. Pagel PS, Kehl F, Gare M, Hettrick DA, Kersten JR, Warltier DC. Mechanical function of the left atrium. New insights based on analysis of pressure-volume relations and Doppler echocardiography. Anesthesiology. 2003;98:975-94.

19. Dernellis JM, Stefanadis CI, Zacharoulis AA, Toutouzas PK. Left atrial mechanical adaptation to long-standing hemodynamic loads based on pressure-volume relations. Am J Cardiol. 1998;81:1138-43.

20. Chien CY, Chen CW, Lin TK, et al. Atrial deformation correlated with functional capacity in mitral stenosis patients. Echocardiography. 2018;35(2):190-5.

21. Trikas A, Papathanasiou S, Tousoulis D, et al. Left atrial function, cytokines and soluble apoptotic markers in mitral stenosis: effects of valvular replacement. Int J Cardiol. 2005;99:111-5.

22. Rohani A, Kargar S, Fazlinejad A, et al. Acute effect of treatment of mitral stenosis on left atrium function. Ann Card Anaesth. 2017;20:42-4.

23. Ancona R, Pinto SC, Caso P, et al. Two-dimensional atrial systolic strain imaging predicts atrial fibrillation at 4-year follow-up in asymptomatic rheumatic mitral stenosis. J Am Soc Echocardiogr. 2013;26:270-7.

24. Vriz O, Blassy B, A Almozel, et al. Left atrial strain can predict right ventricular impairment and development of atrial fibrillation in patients with severe mitral stenosis better than transmitral gradients. Eur Heart J Cardiovasc Imaging. 2021;22:Suppl 1:i70.

25. Mahajan S, Mehra P, Mehta V, et al. Left and right ventricular deformation in patients with severe mitral stenosis and pulmonary hypertension undergoing percutaneous balloon mitral valvuloplasty: A two dimensional speckle-tracking echocardiographic study. Indian Heart J. 2020;72:614-8.

Table 1: Baseline characteristics and echocardiographic parameters among subjects

\begin{tabular}{llll}
\hline & Cases $(\mathrm{n}=80)$ & Controls $(\mathrm{n}=40)$ & $\mathrm{P}$ value \\
\hline Age $($ years $)$ & $30.4 \pm 6.7$ & $29.5 \pm 5.9$ & 0.46 \\
LA size $(\mathrm{cm})$ & $4.67 \pm 0.65$ & $2.79 \pm 0.41$ & $<0.001$ \\
LVID $_{\mathrm{d}}(\mathrm{cm})$ & $4.40 \pm 0.40$ & $4.5 \pm 0.21$ & 0.09 \\
LVID $(\mathrm{cm})$ & $2.79 \pm 0.33$ & $2.68 \pm 0.18$ & 0.06 \\
LVEF $(\%)$ & $59.85 \pm 3.55$ & $60.45 \pm 1.89$ & 0.23 \\
MVA $\left(\mathrm{cm}^{2}\right)$ & $0.93 \pm 0.21$ & $5.17 \pm 0.53$ & $<0.0001$ \\
PG $(\mathrm{mm} \mathrm{Hg})$ & $19.33 \pm 5.82$ & - & -
\end{tabular}




\begin{tabular}{llll}
\hline & Cases $(\mathrm{n}=80)$ & Controls $(\mathrm{n}=40)$ & $\mathrm{P}$ value \\
\hline MG $(\mathrm{mm} \mathrm{Hg})$ & $12.33 \pm 4.16$ & - & - \\
RVSP (mm Hg) & $60.01 \pm 19.88$ & - & - \\
$\mathrm{LAS}_{\mathrm{r}}$ (positive value, \%) & $14.73 \pm 8.59$ & $44.11 \pm 10.44$ & $<0.001$ \\
LAS $_{\text {cd }}$ (negative value, & $-7.61 \pm 4.47$ & $-32.45 \pm 7.63$ & $<0.001$ \\
$\%)$ & $-11.85 \pm 6.77$ & $<0.001$ \\
LAS & & & \\
$\%)$ & $-7.16 \pm 5.15$ & & \\
\hline
\end{tabular}

LA- left atrium; LVEF- left ventricular ejection fraction; LVIDd- left ventricle internal dimension in diastole; LVIDs- left ventricle internal dimension in systole; $\mathrm{LAS}_{\mathrm{r}^{-}}$left atrium reservoir strain; $\mathrm{LAS}_{\mathrm{cd}} \mathrm{l}^{-}$left atrium conduit strain; $\mathrm{LAS}_{\mathrm{ct}}$ - left atrium contractile strain; MG- mean diastolic transmitral gradient; MVA- mitral valve area; PG- peak diastolic transmitral gradient; RVSP- right ventricle systolic pressure

Table 2: Distribution and statistical comparison of echocardiography parameters among patients grouped according to various NYHA classes

\begin{tabular}{|c|c|c|c|c|c|c|c|}
\hline $\begin{array}{l}\text { Echocardiograp } \\
\text { parameters }\end{array}$ & $\begin{array}{l}\text { pAyYHA } \\
\text { class }\end{array}$ & $\begin{array}{l}\text { NYHA } \\
\text { class }\end{array}$ & $\begin{array}{l}\text { NYHA } \\
\text { class }\end{array}$ & $\begin{array}{l}\mathrm{P} \text { value } \\
\text { between } \\
\text { various } \\
\text { NYHA } \\
\text { classes }\end{array}$ & $\begin{array}{l}\mathrm{P} \text { value } \\
\text { between } \\
\text { various } \\
\text { NYHA } \\
\text { classes }\end{array}$ & $\begin{array}{l}\mathrm{P} \text { value } \\
\text { between } \\
\text { various } \\
\text { NYHA } \\
\text { classes }\end{array}$ & $\begin{array}{l}\mathrm{P} \text { value } \\
\text { between } \\
\text { various } \\
\text { NYHA } \\
\text { classes }\end{array}$ \\
\hline & $\begin{array}{l}\mathrm{I}(\text { Mean } \pm \\
\mathrm{SD}, \mathrm{n}=5)\end{array}$ & $\begin{array}{l}\text { II (Mean } \pm \\
\mathrm{SD}, \mathrm{n}=38)\end{array}$ & $\begin{array}{l}\text { III (Mean } \pm \\
\mathrm{SD}, \mathrm{n}=37)\end{array}$ & I and II & II and III & III and I & $\begin{array}{l}\text { Among } \\
\text { three groups }\end{array}$ \\
\hline $\begin{array}{l}\text { LA size } \\
(\mathrm{cm})\end{array}$ & $\begin{array}{l}4.54 \pm \\
0.97\end{array}$ & $\begin{array}{l}4.56 \pm \\
0.57\end{array}$ & $\begin{array}{l}4.81 \pm \\
0.68\end{array}$ & 0.95 & 0.10 & 0.39 & 0.24 \\
\hline $\begin{array}{l}\mathrm{LVID}_{\mathrm{d}} \\
(\mathrm{cm})\end{array}$ & $4.28 \pm 0.3$ & $\begin{array}{l}4.34 \pm \\
0.38\end{array}$ & $\begin{array}{l}4.48 \pm \\
0.43\end{array}$ & 0.75 & 0.13 & 0.29 & 0.25 \\
\hline $\begin{array}{l}\operatorname{LVID}_{\mathrm{s}} \\
(\mathrm{cm})\end{array}$ & $\begin{array}{l}2.68 \pm \\
0.33\end{array}$ & $\begin{array}{l}2.77 \pm \\
0.32\end{array}$ & $\begin{array}{l}2.83 \pm \\
0.36\end{array}$ & 0.57 & 0.47 & 0.36 & 0.58 \\
\hline LVEF (\%) & $\begin{array}{l}58.80 \pm \\
2.86\end{array}$ & $\begin{array}{l}59.87 \pm \\
3.05\end{array}$ & $\begin{array}{l}59.97 \pm \\
4.12\end{array}$ & 0.53 & 0.9 & 0.49 & 0.79 \\
\hline $\begin{array}{l}\text { MVA } \\
\left(\mathrm{cm}^{2}\right)\end{array}$ & $\begin{array}{l}1.18 \pm \\
0.23\end{array}$ & $0.97 \pm 0.2$ & $\begin{array}{l}0.87 \pm \\
0.21\end{array}$ & 0.03 & 0.03 & 0.002 & 0.004 \\
\hline $\begin{array}{l}\mathrm{PG}(\mathrm{mm} \\
\mathrm{Hg})\end{array}$ & $\begin{array}{l}17.40 \pm \\
7.02\end{array}$ & $\begin{array}{l}18.26 \pm \\
5.20\end{array}$ & $\begin{array}{l}20.68 \pm \\
6.13\end{array}$ & 0.75 & 0.07 & 0.24 & 0.15 \\
\hline $\begin{array}{l}\mathrm{MG}(\mathrm{mm} \\
\mathrm{Hg})\end{array}$ & $\begin{array}{l}11.40 \pm \\
5.37\end{array}$ & $\begin{array}{l}11.47 \pm \\
3.55\end{array}$ & $\begin{array}{l}13.32 \pm \\
4.46\end{array}$ & 0.97 & 0.055 & 0.33 & 0.14 \\
\hline $\begin{array}{l}\text { RVSP } \\
(\mathrm{mm} \mathrm{Hg})\end{array}$ & $\begin{array}{l}51.20 \pm \\
21.03\end{array}$ & $\begin{array}{l}55.03 \pm \\
18.47\end{array}$ & $\begin{array}{l}66.32 \pm \\
19.74\end{array}$ & 0.67 & 0.01 & 0.10 & 0.02 \\
\hline $\mathrm{LAS}_{\mathrm{r}}(\%)$ & $\begin{array}{l}14.75 \pm \\
8.98\end{array}$ & $\begin{array}{l}16.53 \pm \\
10.38\end{array}$ & $\begin{array}{l}12.89 \pm \\
5.95\end{array}$ & 0.66 & 0.06 & 0.65 & 0.19 \\
\hline $\mathrm{LAS}_{\mathrm{cd}}(\%)$ & $\begin{array}{l}-7.55 \pm \\
5.61\end{array}$ & $\begin{array}{l}-8.61 \pm \\
5.02\end{array}$ & $\begin{array}{l}-6.58 \pm \\
3.52\end{array}$ & 0.61 & 0.05 & 0.65 & 0.15 \\
\hline $\mathrm{LAS}_{\mathrm{ct}}(\%)$ & $\begin{array}{l}-7.19 \pm \\
6.86\end{array}$ & $\begin{array}{l}-7.87 \pm \\
6.2\end{array}$ & $\begin{array}{l}-6.40 \pm \\
3.54\end{array}$ & 0.78 & 0.22 & 0.75 & 0.48 \\
\hline
\end{tabular}

LA- left atrium; LVEF- left ventricular ejection fraction; LVIDd- left ventricle internal dimension in diastole; LVIDs- left ventricle internal dimension in systole; $\mathrm{LAS}_{\mathrm{r}^{-}}$left atrium reservoir strain; $\mathrm{LAS}_{\mathrm{cd}^{-}}$left atrium 
conduit strain; $\mathrm{LAS}_{\mathrm{ct}}$ - left atrium contractile strain; MG- mean diastolic transmitral gradient; MVA- mitral valve area; PG- peak diastolic transmitral gradient; RVSP- right ventricle systolic pressure

Table 3. Comparison between left atrial strain parameters with various groups based on mean and peak diastolic transmitral gradient, left atrium size, severity of pulmonary hypertension and mitral valve area

\begin{tabular}{|c|c|c|}
\hline Echocardiography parameters & LA strain values & LA strain values \\
\hline & $\mathbf{L A S}_{\mathbf{r}}(\%)$ & $\mathrm{LAS}_{\mathrm{cd}}(\%)$ \\
\hline Mean diastolic transmitral gradient $(\mathrm{mm} \mathrm{Hg})$ & Mean diastolic transmitral gradient $(\mathrm{mm} \mathrm{Hg})$ & Mean diastolic transmitral \\
\hline$<10($ Group I) $\mathrm{n}=26$ & $15.06 \pm 7.74$ & $-8.54 \pm 4.05$ \\
\hline $10-15$ (Group II) $\mathrm{n}=32$ & $15.77 \pm 7.38$ & $-7.90 \pm 4.14$ \\
\hline $15-20$ (Group III) $\mathrm{n}=15$ & $13.83 \pm 12.32$ & $-6.68 \pm 5.76$ \\
\hline$>20($ Group IV $) \mathrm{n}=7$ & $10.77 \pm 7.69$ & $-4.80 \pm 3.63$ \\
\hline Peak diastolic transmitral gradient ( $\mathrm{mm} \mathrm{Hg}$ ) & Peak diastolic transmitral gradient (mm Hg) & Peak diastolic transmitral \\
\hline $10-20($ Group I) $\mathrm{n}=50$ & $15.20 \pm 7.89$ & $-8.12 \pm 4.26$ \\
\hline $20-30$ (Group II) $n=25$ & $14.09 \pm 10.13$ & $-6.91 \pm 4.90$ \\
\hline$>30($ Group III $) \mathrm{n}=5$ & $13.17 \pm 8.42$ & $-5.91 \pm 4.27$ \\
\hline Left atrium size $(\mathrm{cm})$ & Left atrium size $(\mathrm{cm})$ & Left atrium size $(\mathrm{cm})$ \\
\hline$<4($ Group I) $n=11$ & $14.35 \pm 7.15$ & $-9.38 \pm 4.59$ \\
\hline $4-5$ (Group II) $n=44$ & $15.41 \pm 8.17$ & $-7.70 \pm 4.22$ \\
\hline $5-6$ (Group III) $n=20$ & $15.10 \pm 10.70$ & $-7.31 \pm 5.08$ \\
\hline$>6($ Group IV) $\mathrm{n}=5$ & $8.11 \pm 2.59$ & $-4.02 \pm 1.70$ \\
\hline Severity of pulmonary hypertension & Severity of pulmonary hypertension & Severity of pulmonary hyl \\
\hline No $($ Group I) $n=6$ & $19.71 \pm 11.67$ & $-9.08 \pm 5.06$ \\
\hline Mild (Group II) $n=18$ & $15.22 \pm 6.67$ & $-8.67 \pm 4.44$ \\
\hline Moderate (Group III) $\mathrm{n}=19$ & $15.37 \pm 10.71$ & $-8.01 \pm 5.18$ \\
\hline Severe (Group IV) $\mathrm{n}=37$ & $13.36 \pm 7.64$ & $-6.64 \pm 3.95$ \\
\hline Mitral valve area & Mitral valve area & Mitral valve area \\
\hline$<1 \mathrm{~cm}^{2}($ Group I) $\mathrm{n}=44$ & $15.60 \pm 9.90$ & $-7.87 \pm 5.07$ \\
\hline $1-1.5 \mathrm{~cm}^{2}$ (Group II) $\mathrm{n}=36$ & $13.67 \pm 6.63$ & $-7.28 \pm 3.65$ \\
\hline
\end{tabular}

$\mathrm{LAS}_{\mathrm{r}}$ - left atrium reservoir strain; $\mathrm{LAS}_{\mathrm{cd}}$ - left atrium conduit strain; $\mathrm{LAS}_{\mathrm{ct}}$ - left atrium contractile strain

Table 4. Statistical intergroup significance between left atrial strain parameters with various groups based on mean and peak diastolic transmitral gradient, left atrium size, severity of pulmonary hypertension and mitral valve area

Left atrium strain parameters

$\mathrm{LAS}_{\mathrm{r}}$

LAS $_{\mathrm{cd}}$

LAS $_{\mathrm{ct}}$

$\mathrm{P}$ value between four groups based on mean diastolic transmitral gradient

$\mathrm{LAS}_{\mathrm{r}}$

$\mathrm{LAS}_{\mathrm{cd}}$

LAS $_{\text {ct }}$

$\mathrm{P}$ value between four groups based on severity of pulmonary hypertension

$\mathrm{LAS}_{\mathrm{r}}$

LAS $_{c d}$

$\mathrm{LAS}_{\mathrm{ct}}$
Left atrium strain parameters

$\mathrm{LAS}_{\mathrm{r}}$

$\mathrm{LAS}_{\mathrm{cd}}$

$\mathrm{LAS}_{\mathrm{ct}}$

$P$ value between four groups based on mean di

$\mathrm{LAS}_{\mathrm{r}}$

$\mathrm{LAS}_{\mathrm{cd}}$

$\mathrm{LAS}_{\mathrm{ct}}$

$\mathrm{P}$ value between four groups based on severity

$\mathrm{LAS}_{\mathrm{r}}$

$\mathrm{LAS}_{\mathrm{cd}}$

$\mathrm{LAS}_{\mathrm{ct}}$ 
Left atrium strain parameters

$\mathrm{P}$ value between three groups based on peak diastolic transmitral gradient $\mathrm{LAS}_{\mathrm{r}}$

$\mathrm{LAS}_{\mathrm{cd}}$

$\mathrm{LAS}_{\mathrm{ct}}$

$\mathrm{P}$ value between two groups based on mitral valve area

$\mathrm{LAS}_{\mathrm{r}}$

$\mathrm{LAS}_{\mathrm{cd}}$

$\mathrm{LAS}_{\mathrm{ct}}$
Left atrium strain parameters

$P$ value between three groups based on peak $d$ LAS $_{\mathrm{r}}$

$\mathrm{LAS}_{\mathrm{cd}}$

$\mathrm{LAS}_{\mathrm{ct}}$

$\mathrm{P}$ value between two groups based on mitral vi $\mathrm{LAS}_{\mathrm{r}}$

$\mathrm{LAS}_{\mathrm{cd}}$

$\mathrm{LAS}_{\mathrm{ct}}$

$\mathrm{LAS}_{\mathrm{r}}$ - left atrium reservoir strain; $\mathrm{LAS}_{\mathrm{cd}}$ - left atrium conduit strain; $\mathrm{LAS}_{\mathrm{ct}}$ - left atrium contractile strain

Figure Legend

Figure 1: Left atrial two dimensional speckle tracking echocardiography strain (reservoir, conduit and contractile strain) values in end-diastole, (A) healthy control and (B) patient with severe mitral stenosis. LASr- left atrium reservoir strain; LAScd- left atrium conduit strain; LASct- left atrium contractile strain

\section{Table Legends}

Table 1: Baseline characteristics and echocardiographic parameters among subjects

Table 2: Distribution and statistical comparison of echocardiography parameters among patients grouped according to various NYHA classes

Table 3. Comparison between left atrial strain parameters with various groups based on mean and peak diastolic transmitral gradient, left atrium size, severity of pulmonary hypertension and mitral valve area

Table 4. Statistical intergroup significance between left atrial strain parameters with various groups based on mean and peak diastolic transmitral gradient, left atrium size, severity of pulmonary hypertension and mitral valve area 


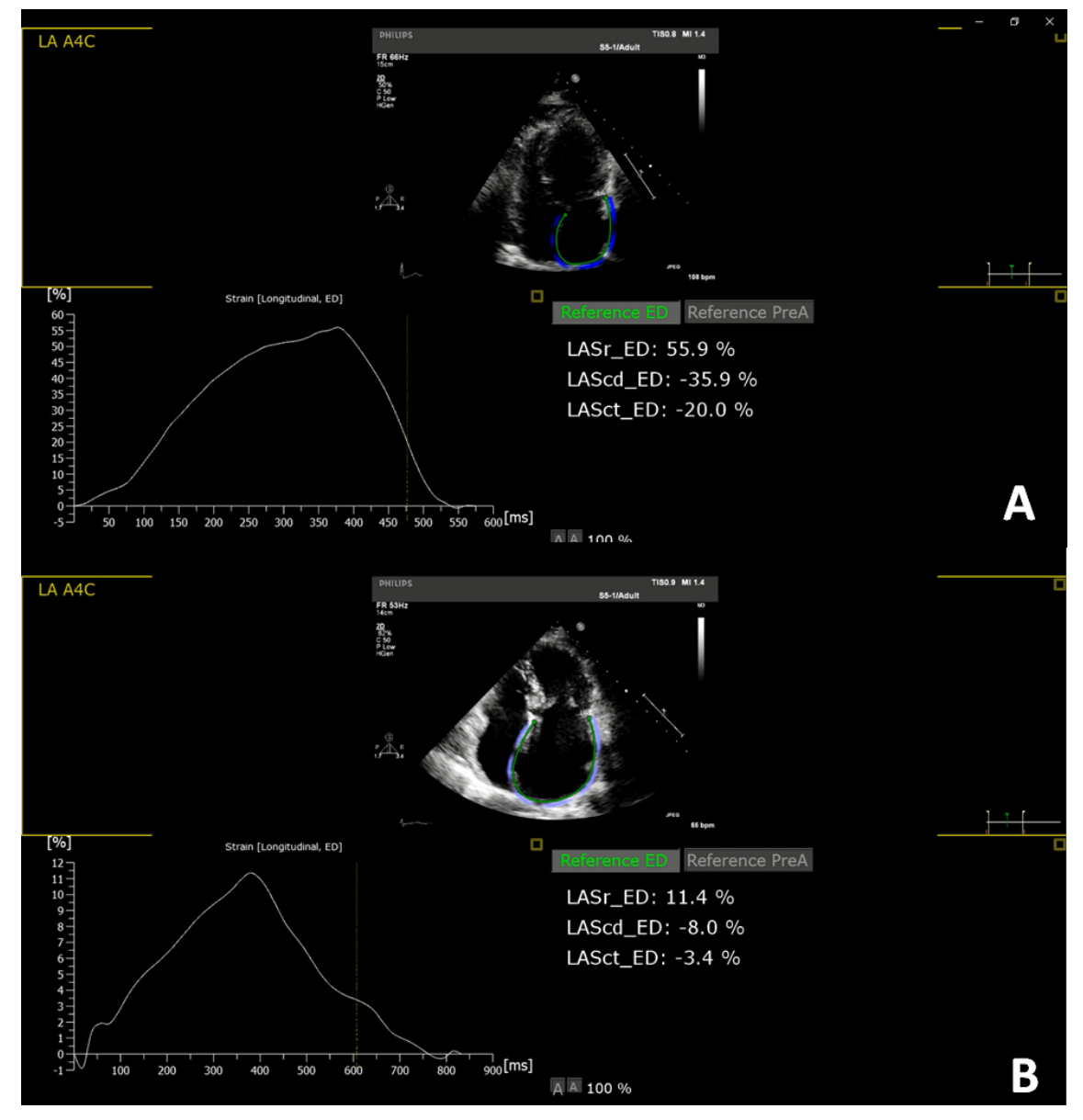

